\title{
Epigenetic chromatin modification by amber suppression technology
}

Heinz Neumann' ${ }^{1}$ Petra Neumann-Staubitz ${ }^{1}$, Anna Witte ${ }^{2}$ and Daniel Summerer ${ }^{2}$

${ }^{1}$ Max-Planck-Institute for Molecular Physiology

Otto-Hahn-Strasse 11

44227 Dortmund, Germany

${ }^{2}$ Faculty of Chemistry and Chemical Biology

TU Dortmund University

Otto-Hahn-Str. 4a, 44227 Dortmund

Correspondence: heinz.neumann@mpi-dortmund.mpg.de,daniel.summerer@tu-dortmund.de

Keywords: Genetic Code Expansion; Unnatural Amino Acids; UV-crosslinking; Post-translational modifications; Bioorthogonal Chemistry 


\section{Abstract}

The genetic incorporation of unnatural amino acids (UAAs) into proteins by amber suppression technology provides unique avenues to study protein structure, function and interactions both in vitro and in living cells and organisms. This approach has been particularly useful for studying mechanisms of epigenetic chromatin regulation, since these extensively involve dynamic changes in structure, complex formation and posttranslational modifications that are difficult to access by traditional approaches. Here, we review recent achievements in this field, emphasizing UAAs that help to unravel protein-protein interactions in cells by photo-crosslinking or that allow the biosynthesis of proteins with defined posttranslational modifications for studying their function and turnover in vitro and in cells. 


\section{Introduction}

The incorporation of UAAs, in response to nonsense codons (such as the amber stop codon, UAG) enables the biosynthesis of proteins with unique properties (Fig. 1A). This is achieved by the heterologous expression of an orthogonal aminoacyl-tRNA synthetase (aaRS)/tRNA ${ }_{\text {CUA }}$ pair in the host organism. The aaRS of this pair is evolved to charge an UAA of choice to its cognate tRNA, which in turn is engineered to direct the incorporation of this UAA in response to amber codons (Fig. 1A). With this approach, a plethora of useful UAAs has been added to the genetic code of various organisms ranging from prokaryotes and yeasts to entire animals and plants [1]. The neo-functionalised proteins are being employed in a wide range of applications [2], with studies on epigenetic mechanisms of chromatin regulation being a particular focus. In the following, we review the most recent studies in this field, dealing with elucidating protein-protein interactions with photo-crosslinking UAAs and studying the function of posttranslational modifications (PTMs) by their direct incorporation as UAAs.

\section{Photo-crosslinkers}

The incorporation of photo-crosslinker UAAs at defined positions of a protein offers the opportunity to trap its interactions with other macromolecules. Different types of crosslinker UAAs have been successfully introduced into proteins of pro- and eukaryotes [3]. The most popular choice is p-benzoylphenylalanine (BPA) because of its high crosslinking yield and commercial availability. Aromatic azides and diazirines have also extensively been used for crosslinking. These compounds are less bulky and hydrophobic than BPA and therefore have a lower potential to disturb the activity of the mutated protein. Applications of crosslinker UAAs in epigenetics range from the study of static binary interactions in vitro to the relative quantification of protein-protein interactions in living cells.

\section{$\underline{\text { In vitro crosslinking }}$}

Recognition of $\mathrm{H} 3 \mathrm{~K} 9 \mathrm{me} 3$ by HP1 is required for heterochromatin formation [4,5]. How this interaction drives heterochromatinization of genomic loci is, however, still controversial. Using BPA incorporation in both the $\mathrm{H} 3 \mathrm{~K} 9 \mathrm{me} 3$ reading chromodomain and the dimerization mediating HP1 chromoshadow 
domain, it was shown that HP1 bridges fibres in condensed chromatin, thereby stabilizing the compacted state [6].

Chromatin compaction is also directly influenced by PTMs. H2B ubiquitination interferes with fibre compaction by acting as a 'wedge' between neighbouring nucleosomes. Fibre compaction also induces ubiquitin-ubiquitin contacts, as shown by BPA-crosslinking, impairing oligomerization [7].

The identification of the crosslinked peptide by mass spectrometry provides additional structural information. The challenge lies in the complex fragmentation pattern these peptides generate, limiting the scope of this approach to well-defined systems. Incorporation of BPA in chromatin remodeler ISWI provided insights into the function of its motor domain and into its regulation by $\mathrm{H} 4$-tail binding $[8,9]$.

\section{In vivo crosslinking}

A particularly powerful application of crosslinker UAAs is to study protein-protein interactions in living cells. The combination of site-specific incorporation and photo-activation provides precise control over the reaction and is particularly useful to trap transient interactions and to map interaction surfaces. The approach has been used to study the interaction of histone chaperones Asf1 [10] and FACT [11] with their substrates, and to reveal interactions of transcription factors with the Swi/Snf chromatin remodelling complex [12] in yeast.

H2B ubiquitination is deposited by the Paf1 complex during transcription. How exactly the complex recruits the ubiquitination machinery was studied by BPA-crosslinking in live yeast (Fig. 1B) [13*]. The Rtf1 subunit of the Paf1 complex was shown to directly interact with ubiquitin conjugase Rad6 via a conserved surface of its histone modification domain (HMD). This report demonstrates the power of BPA-crosslinking because standard methods did not detect this transient interaction reliably. Furthermore, the crosslink reaction can be used to assay the dependence of an interaction on additional factors. In this case, deletion of BRE1, the corresponding ubiquitin ligase, did not affect Rtf1HMD-Rad6 crosslinking but reduced crosslinking of full-length Rtf1 to Rad6. This suggests that Bre1 regulates the Rtf1-Rad6 interaction indirectly via other subunits of the Paf1 complex. 
Photo-crosslinking with UAAs can also be used to quantify interactions at different stages of a synchronized process. The interaction of the $\mathrm{H} 4 \mathrm{~N}$-terminal tail with the acidic patch on neighbouring nucleosomes was shown to peak in mitosis using BPA-crosslinking in cell cycle synchronized yeast [14]. Mutational studies, using the crosslink reaction as a readout, identified a signalling cascade that controlled the interaction. This interaction contributes to the compaction of chromosomes in mitosis independently of condensins $[14,15]$.

\section{Method development}

The relative magnitude of the same crosslink reaction can be used to quantify interactions in different contexts such as cell cycle stages or strain backgrounds. Crosslinking efficiencies, however, vary greatly between different positions of UAA incorporation and are thus not a measure of affinities. The absence of an expected crosslink is no evidence against an interaction. Sometimes alternative crosslinkers produce different crosslink patterns. The influence of sequence context on crosslinking efficiency has been studied [16,17], revealing a strong preference of BPA for methionine residues, representing a potential source of quenching.

The identification of reader proteins for $\mathrm{N} \varepsilon$-crotonyl-lysine has been facilitated by functionalizing its $\gamma$ carbon with a diazirine group to create a photo-affinity analogue (i, Fig. 1C) [18*]. Identification of crosslinked proteins is usually the limiting step in the analysis. This can be achieved by comparing crosslink reactions in two different strain backgrounds differing in the presence of an epitope tag on the candidate protein that induces a mobility shift in Western blots. An unbiased identification of crosslink products requires their isolation for analysis by mass spectrometry. This is often challenging but has been achieved, e.g., for interactors of transcription factors in budding yeast [19*] and histone $\mathrm{H} 3$ and $\mathrm{H} 4$ in mammalian cells [20*].

An even greater challenge, that usually requires the isolation of microgram quantities of crosslinked material, is the identification of the crosslinked peptide itself and has so far only been mastered with purified components. The development of bifunctional crosslinker UAAs with an additional 
bioorthogonal handle for enrichment of the crosslinked peptide (ii, Fig. 1C) [21] or a chemically cleavable crosslinker (iii) $[22,23]$ may facilitate such analyses with material from eukaryotic sources. BPA-crosslinking has been combined with chemical crosslinking by formaldehyde [24]. This improved the crosslinking efficiency of transient interactions and could also be used to simultaneously localise the interaction on the genome.

\section{Posttranslational modifications}

Posttranslational modifications (PTMs) of histones extensively regulate the condensation state of chromatin and thus its function in transcription, replication, and DNA repair [25]. PTMs can act alone, but often multiple distinct PTMs on one or more histone tails act combinatorially to trigger distinct downstream events [26]. Studying the roles of PTMs has been hampered by a limited synthetic access to proteins with defined PTM patterns, however, chemical modification strategies such as ligation methods [27] and amber suppression [28] have already made significant contributions to resolve this bottleneck.

\section{Lysine acetylation}

$N \varepsilon$-acetyl-lysine (AcK, Fig. 2A) is a key regulatory PTM abundantly found in all histones $[25,26]$. Sitespecific incorporation of AcK by amber suppression has been achieved with an evolved $M$. barkeri PyIRS/tRNA ${ }^{\text {Pyl }}($ CUA) pair in E. coli [29] and stable mammalian cell lines [30**], and in a first study revealed effects of $\mathrm{H} 3 \mathrm{~K} 56$ acetylation on nucleosome breathing in in vitro FRET experiments [31]. Since then, Ack has extensively been used to study the role of lysine acetylation in histones and other proteins. For example, interactions of the PHD/BRD/PWWP reader cassette of ZMYND8 with acetylated $\mathrm{H} 3$ and $\mathrm{H} 4$ peptides were studied in vitro, highlighting the importance of the BRD domain [32].

Moreover, AcK was used in the context of reconstituted nucleosomes to study the role of H3K56Ac and $\mathrm{H} 3 \mathrm{~K} 14 \mathrm{Ac}$ in base excision repair, i.e. the removal of deaminated cytosines and filling-in of single 
nucleotide gaps. Acetylation decreased the activity of DNA polymerase $\beta$ at both positions, without observable effect on nucleosome structure or dynamics [33].

In a further study, acetylation of kinase Aurora B was studied in view of its role in chromosome biorientation during mitosis. Aurora $B$ is part of the chromosomal passenger complex (CPC), which governs chromosome segregation by generating spindle checkpoint signals and correcting aberrant kinetochore-microtubule attachments. Aurora B can be activated by phosphorylation of T232 in its activation loop, which can be reversed by dephosphorylation via phosphatase PP2A. Acetylation of the single residue K215 close to the activation loop by acetyltransferase TIP60 was discovered to protect T232 from this dephosphorylation and thus stabilize the active form. TIP60 itself is regulated via phosphorylation by the cyclin-dependent kinase 1 (CDK1)-cyclin B complex, linking it to robust, errorfree metaphase-anaphase transition via a phosphorylation/acetylation signalling axis [34*] (Fig. 2B).

Amber suppression with AcK has further been utilized to study the activity and substrate scope of histone deacetylases of the sirtuin class. For example, testing a larger set of AcK sites in histone $\mathrm{H} 3$, SIRT1 and 2 were shown to exhibit lower substrate preferences on reconstituted nucleosomes in vitro than expected from in vivo studies [35]. Moreover, the preference of SIRT6 to deacylate lysines bearing long nonpolar acyl groups was exploited to study its histone substrate selectivity via a fluorescence assay based on incorporation of the reporter amino acid $\mathrm{N} \varepsilon$-(7-octenoyl)-lysine (OcK) that can be conjugated to tetrazin-fluorophores [36].

Recently, AcK has even been used to develop fluorescent probes even for the intracellular detection of histone deacetylase activity [37**]. Incorporation of AcK at a single lysine position in GFP (and mCherry) was found to block chromophore maturation in E. coli. This position was found to be a substrate of the endogenous $E$. coli deacetylase cobB, allowing it to trigger maturation and formation of a fluorescence signal (Fig. 2C). This strategy was transferrable to several mammalian sirtuins expressed in a $\triangle$ cobB E. coli strain. Moreover, when the probe was expressed in a mammalian cell line, fluorescence was observed in the presence of classical $\mathrm{Zn}^{2+}$-dependent histone deacetylase inhibitors, 
but not sirtuin inhibitors. Interestingly, incorporation of the PTM NE-2-hydroxyisobutyryl-lysine (HibK, Fig. 3A) [38] by amber suppression $[39,40]$ afforded a functional probe that revealed cobB, SIRT1 and SIRT2 but no other sirtuin as erasers of HibK $\left[37^{* *}\right]$.

\section{Other lysine PTMs}

Besides AcK and HibK, the growing number of alternative lysine acylation types found in histones [41] has led to the development of strategies for their incorporation into proteins by amber suppression. Using engineered PylRS/tRNA ${ }^{\text {Pyl }}$ pairs, Ne-propionyllysine (PrK), Ne-butyryllysine (BuK) and $N \varepsilon$ crotonyllysine (CrK) (Fig. 3A) were the first PTMs to be incorporated into histones and other proteins [42-44] including histone $\mathrm{H} 4$ [45]. Moreover, the genetic encoding of $\mathrm{N} \varepsilon$-formyllysine (Fork [46], Fig. 3A) was reported later [47]. Incorporation of CrK (and other acyllysines) into H3K14 was used to study the recognition scope of the double PHD Finger (DPF) reader domains of MOZ and DPF2, revealing that both can accommodate a wide range of acylations at H3K14 with the strongest preference for CrK. Crystal structural analysis of MOZ DPF showed accommodation of CrK in a tight hydrophobic pocket assisted by water-mediated recognition of the amide group (Fig. 3B) [48].

Besides strategies for direct incorporation, a versatile approach for the two-step in vitro installation of various acylation types on lysine was reported. Azidonorleucine was incorporated into proteins using an evolved PyIRS mutant followed by its selective modification via a traceless Staudinger ligation. Various acyl substituents on the phosphinothioester reactant were successfully used, enabling the installation of all aforementioned acylation types on proteins. Moreover, larger and negatively charged acyl substituents could be installed for that no PyIRS mutants are available, such as malonyl-, succinyl, glutaryl-, and biotinyl-substituents (Fig. 3C) [49*]. Another indirect strategy provided a new access to NE-dimethyllysine-modified histones, a PTM that could previously only be installed under denaturing conditions [50]. Ne-(4-azidobenzyloxycarbonyl)- $\delta, \varepsilon$-dehydrolysine was incorporated and the azido group in the purified protein reduced with TCEP, leading to formation of $\delta, \varepsilon$-dehydrolysine and 
subsequent hydrolysis of its enamine moiety. The resulting aldehyde was then reacted with dimethylamine in a reductive amination, yielding the desired PTM [51].

\section{Phosphorylation}

Significant progress has also been made in the genetic encoding of phosphorylated amino acids. Building on previous studies on incorporation of phosphoserine (pS) using the archaeal SepRS/tRNA ${ }^{\text {Cys }}$ pair capable of charging pS to tRNA ${ }^{\text {Cys }}[52,53]$, redesign of the CUA-mutated tRNA anticodon loop and its recognition by SepRS led to increased incorporation efficiencies of pS and allowed incorporation of a nonhydrolyzable analogue after downregulation of pS biosynthesis [54]. Using this approach, twenty differently linked phosphoubiquitin dimers bearing pS at three different positions were synthesized, enabling insights into the role of phosphorylation on the linkage selectivity of E3 ligase UBE3C and of a large number of deubiquitinases [55*]. Beyond pS, also phosphothreonine (including its biosynthesis) $\left[56^{* *}\right]$ and phosphotyrosine (together with a nonhydrolyzable analogue) [57**] were genetically encoded in E. coli recently.

\section{Photo-activation}

A powerful approach to follow the temporal progression of a physiological process within a living cell is to introduce photocaged amino acids at essential residues in the participating proteins. For example, in many cancers the key arginine in the active centre of the citric acid cycle enzyme Isocitrate dehydrogenase $2(\mathrm{IDH} 2)$ is mutated to lysine, resulting in high levels of the oncometabolite (R)-2hydroxyglutarate $(2 \mathrm{HG})$ contributing to cellular transformation (Fig. 4). By replacing the arginine with a photocaged lysine the Chin group measured a rapid accumulation 2 HG followed by an increase of 5hydroxymethylcytosine levels upon enzyme activation, which may contribute to an epigenetic imbalance and cell transformation [58*]. Thus, amber suppression has yet an extendable application in mammalian cells if temporally defined sequences of events, metabolic and epigenetic changes are in the focus of interest. 


\section{Outlook}

The large number of UAA-based studies and the associated discoveries in chromatin biology illustrate the growing impact of amber suppression technologies on this field. We expect that this impact will keep increasing as amber suppression technologies become standard tools. The further development of amber suppression in mammalian cell lines and animals promises great opportunities to study chromatin dynamics and epigenetic phenomena at this level. Particularly the combination of this technology with other methods such as proteomics and DNA sequencing-based high-throughput methods will greatly enrich the tool kit of chromatin research in the future.

\section{Acknowledgements}

The authors are grateful for financial support by the Heisenberg Program of the German Research Foundation (DFG) [NE1589/5-1 to H.N.] and by project Su 726/6-1 funded within SPP1623 of the DFG [D.S.].

\section{References}

1. Chin JW: Expanding and reprogramming the genetic code of cells and animals. Annu Rev Biochem 2014, 83:379-408.

2. Neumann-Staubitz $P$, Neumann $H$ : The use of unnatural amino acids to study and engineer protein function. Curr Opin Struct Biol 2016, 38:119-128.

3. Yang $Y$, Song $H$, Chen PR: Genetically encoded photocrosslinkers for identifying and mapping protein-protein interactions in living cells. IUBMB Life 2016, 68:879-886.

4. Jacobs SA, Khorasanizadeh S: Structure of HP1 chromodomain bound to a lysine 9-methylated histone $\mathrm{H3}$ tail. Science 2002, 295:2080-2083.

5. Nielsen PR, Nietlispach D, Mott HR, Callaghan J, Bannister A, Kouzarides T, Murzin AG, Murzina NV, Laue ED: Structure of the HP1 chromodomain bound to histone H3 methylated at lysine 9. Nature 2002, 416:103-107.

6. Hiragami-Hamada K, Soeroes S, Nikolov M, Wilkins B, Kreuz S, Chen C, De La Rosa-Velazquez IA, Zenn HM, Kost N, Pohl W, et al.: Dynamic and flexible H3K9me3 bridging via HP1beta dimerization establishes a plastic state of condensed chromatin. Nat Commun 2016, 7:11310.

7. Debelouchina GT, Gerecht K, Muir TW: Ubiquitin utilizes an acidic surface patch to alter chromatin structure. Nat Chem Biol 2017, 13:105-110.

8. Forne I, Ludwigsen J, Imh of A, Becker PB, Mueller-Planitz F: Probing the conformation of the ISWI ATPase domain with genetically encoded photoreactive crosslinkers and mass spectrometry. Mol Cell Proteomics 2012, 11:M111 012088.

9. Ludwigsen J, Pfennig S, Singh AK, Schindler C, Harrer N, Forne I, Zacharias M, Mueller-Planitz F: Concerted regulation of ISWI by an autoinhibitory domain and the $\mathrm{H} 4 \mathrm{~N}$-terminal tail. Elife 2017, 6 . 
10. Dennehey BK, Noone S, Liu WH, Smith L, Churchill ME, Tyler JK: The C terminus of the histone chaperone Asf1 cross-links to histone $\mathrm{H} 3$ in yeast and promotes interaction with histones H3 and H4. Mol Cell Biol 2013, 33:605-621.

11. Hoffmann C, Neumann H: In Vivo Mapping of FACT-Histone Interactions Identifies a Role of Pob3 C-terminus in H2A-H2B Binding. ACS Chem Biol 2015, 10:2753-2763.

12. Krishnamurthy M, Dugan A, Nwokoye A, Fung YH, Lancia JK, Majmudar CY, Mapp AK: Caught in the act: covalent cross-linking captures activator-coactivator interactions in vivo. ACS Chem Biol 2011, 6:1321-1326.

13.* Van Oss SB, Shirra MK, Bataille AR, Wier AD, Yen K, Vinayachandran V, Byeon IL, Cucinotta CE, Heroux A, Jeon J, et al.: The Histone Modification Domain of Paf1 Complex Subunit Rtf1 Directly Stimulates H2B Ubiquitylation through an Interaction with Rad6. Mol Cell 2016, 64:815-825.

BPA-crosslinking in yeast was used to trap a transient interaction of the Paf1C-subunit Rtf1 with E2 ubiquitin conjugase Rad6.

14. Wilkins BJ, Rall NA, Ostwal Y, Kruitwagen T, Hiragami-Hamada K, Winkler M, Barral Y, Fischle W, Neumann $\mathrm{H}$ : A cascade of histone modifications induces chromatin condensation in mitosis. Science 2014, 343:77-80.

15. Kruitwagen T, Denoth-Lippuner A, Wilkins BJ, Neumann H, Barral Y: Axial contraction and shortrange compaction of chromatin synergistically promote mitotic chromosome condensation. Elife 2015, 4:e1039.

16. Lancia JK, Nwokoye A, Dugan A, Joiner C, Pricer R, Mapp AK: Sequence context and crosslinking mechanism affect the efficiency of in vivo capture of a protein-protein interaction. Biopolymers 2014, 101:391-397.

17. Wittelsberger A, Thomas BE, Mierke DF, Rosenblatt M: Methionine acts as a "magnet" in photoaffinity crosslinking experiments. FEBS Lett 2006, 580:1872-1876.

18.* Xie X, Li XM, Qin F, Lin J, Zhang G, Zhao J, Bao X, Zhu R, Song H, Li XD, et al.: Genetically Encoded Photoaffinity Histone Marks. J Am Chem Soc 2017, 139:6522-6525.

Crotonyl-lysine modified with a diazirine function at the $\gamma$-carbon was introduced into histones to trap reader and eraser proteins of this modification.

19.* Dugan A, Majmudar CY, Pricer R, Niessen S, Lancia JK, Fung HY, Cravatt BF, Mapp AK: Discovery of Enzymatic Targets of Transcriptional Activators via in Vivo Covalent Chemical Capture. $J$ Am Chem Soc 2016, 138:12629-12635.

The authors coupled in vivo covalent chemical capture and shotgun LC-MS/MS to identify interaction partners of transcriptional activators in budding yeast.

20.* Kleiner RE, Hang LE, Molloy KR, Chait BT, Kapoor TM: A Chemical Proteomics Approach to Reveal Direct Protein-Protein Interactions in Living Cells. Cell Chem Biol 2017.

The study investigates interactions of histone $\mathrm{H} 3$ and $\mathrm{H} 4$ tails in vivo using a genetically encoded diazirine derivative of lysine.

21. Joiner CM, Breen ME, Clayton J, Mapp AK: A Bifunctional Amino Acid Enables Both Covalent Chemical Capture and Isolation of in Vivo Protein-Protein Interactions. Chembiochem 2017, 18:181-184.

22. Lin S, He D, Long T, Zhang S, Meng R, Chen PR: Genetically encoded cleavable protein photocross-linker. J Am Chem Soc 2014, 136:11860-11863. 
23. Yang $Y$, Song H, He D, Zhang S, Dai S, Xie X, Lin S, Hao Z, Zheng H, Chen PR: Genetically encoded releasable photo-cross-linking strategies for studying protein-protein interactions in living cells. Nat Protoc 2017, 12:2147-2168.

24. Dugan A, Pricer R, Katz M, Mapp AK: TRIC: Capturing the direct cellular targets of promoterbound transcriptional activators. Protein Sci 2016, 25:1371-1377.

25. Kouzarides T: Chromatin modifications and their function. Cell 2007, 128:693-705.

26. Jenuwein T, Allis CD: Translating the histone code. Science 2001, 293:1074-1080.

27. Muller MM, Muir TW: Histones: at the crossroads of peptide and protein chemistry. Chem Rev 2015, 115:2296-2349.

28. Chin JW: Expanding and reprogramming the genetic code. Nature 2017, 550:53-60.

29. Neumann H, Peak-Chew SY, Chin JW: Genetically encoding N(epsilon)-acetyllysine in recombinant proteins. Nat Chem Biol 2008, 4:232-234.

30.** Elsasser SJ, Ernst RJ, Walker OS, Chin JW: Genetic code expansion in stable cell lines enables encoded chromatin modification. Nat Methods 2016, 13:158-164.

The study reports the genetic encoding of e.g. AcK in cell lines stably expressing the orthogonal aaRS/tRNA pair and thus represents a basis for studying long-term processes.

31. Neumann H, Hancock SM, Buning R, Routh A, Chapman L, Somers J, Owen-Hughes T, van Noort J, Rhodes D, Chin JW: A method for genetically installing site-specific acetylation in recombinant histones defines the effects of H3 K56 acetylation. Mol Cell 2009, 36:153-163.

32. Savitsky P, Krojer T, Fujisawa T, Lambert JP, Picaud S, Wang CY, Shanle EK, Krajewski K, Friedrichsen $\mathrm{H}$, Kanapin $\mathrm{A}$, et al.: Multivalent Histone and DNA Engagement by a PHD/BRD/PWWP Triple Reader Cassette Recruits ZMYND8 to K14ac-Rich Chromatin. Cell Reports 2016, 17:2724-2737.

33. Rodriguez Y, Hinz JM, Laughery MF, Wyrick JJ, Smerdon MJ: Site-specific Acetylation of Histone H3 Decreases Polymerase Activity on Nucleosome Core Particles in Vitro. Journal of Biological Chemistry 2016, 291:11434-11445.

34.* Mo F, Zhuang X, Liu X, Yao PY, Qin B, Su Z, Zang J, Wang Z, Zhang J, Dou Z, et al.: Acetylation of Aurora B by TIP60 ensures accurate chromosomal segregation. Nat Chem Biol 2016, 12:226232.

This study provides simple and versatile methodology to detect sirtuin activity in live cells.

35. Hsu WW, Wu B, Liu WSR: Sirtuins 1 and 2 Are Universal Histone Deacetylases. Acs Chemical Biology 2016, 11:792-799.

36. Wang WW, Zeng Y, Wu B, Deiters A, Liu WR: A Chemical Biology Approach to Reveal Sirt6targeted Histone H3 Sites in Nucleosomes. Acs Chemical Biology 2016, 11:1973-1981.

37.** Xuan WM, Yao AZ, Schultz PG: Genetically Encoded Fluorescent Probe for Detecting Sirtuins in Living Cells. Journal of the American Chemical Society 2017, 139:12350-12353.

This study provides simple and versatile methodology to detect sirtuin activity in live cells.

38. Dai L, Peng C, Montellier E, Lu Z, Chen Y, Ishii H, Debernardi A, Buchou T, Rousseaux S, Jin F, et al.: Lysine 2-hydroxyisobutyrylation is a widely distributed active histone mark. Nat Chem Biol 2014, 10:365-370.

39. Xiao H, Xuan WM, Shao SD, Liu T, Schultz PG: Genetic Incorporation of epsilon-N-2Hydroxyisobutyryl-lysine into Recombinant Histones. Acs Chemical Biology 2015, 10:15991603.

40. Knight WA, Cropp TA: Genetic encoding of the post-translational modification 2hydroxyisobutyryl-lysine. Organic \& Biomolecular Chemistry 2015, 13:6479-6481.

41. Sabari $B R$, Zhang $D$, Allis $C D$, Zhao Y: Metabolic regulation of gene expression through histone acylations. Nat Rev Mol Cell Biol 2017, 18:90-101. 
42. Gattner MJ, Vrabel M, Carell T: Synthesis of epsilon-N-propionyl-, epsilon-N-butyryl-, and epsilon-N-crotonyl-lysine containing histone $\mathrm{H} 3$ using the pyrrolysine system. Chem Commun (Camb) 2013, 49:379-381.

43. Kim CH, Kang M, Kim HJ, Chatterjee A, Schultz PG: Site-specific incorporation of epsilon-Ncrotonyllysine into histones. Angew Chem Int Ed Eng/ 2012, 51:7246-7249.

44. Lee YJ, Wu B, Raymond JE, Zeng Y, Fang X, Wooley KL, Liu WR: A genetically encoded acrylamide functionality. ACS Chem Biol 2013, 8:1664-1670.

45. Wilkins BJ, Hahn LE, Heitmuller S, Frauendorf H, Valerius O, Braus GH, Neumann H: Genetically Encoding Lysine Modifications on Histone H4. ACS Chemical Biology 2015, 10:939-944.

46. Wisniewski JR, Zougman A, Mann M: Nepsilon-formylation of lysine is a widespread posttranslational modification of nuclear proteins occurring at residues involved in regulation of chromatin function. Nucleic Acids Res 2008, 36:570-577.

47. Wang TY, Zhou Q, Li FH, Yu Y, Yin XB, Wang JY: Genetic Incorporation of N-epsilon-Formyllysine, a New Histone Post-translational Modification. Chembiochem 2015, 16:1440-1442.

48. Xiong $X$, Panchenko T, Yang S, Zhao S, Yan P, Zhang W, Xie W, Li Y, Zhao Y, Allis CD, et al.: Selective recognition of histone crotonylation by double PHD fingers of $\mathrm{MOZ}$ and DPF2. Nat Chem Biol 2016, 12:1111-1118.

49.* Wang ZPA, Kurra Y, Wang X, Zeng Y, Lee YJ, Sharma V, Lin HN, Dai SY, Liu WSR: A Versatile Approach for Site-Specific Lysine Acylation in Proteins. Angewandte Chemie-International Edition 2017, 56:1643-1647.

The study significantly expands the scope of acylation types that can be introduced into proteins for in vitro studies.

50. Nguyen DP, Garcia Alai MM, Virdee S, Chin JW: Genetically directing varepsilon-N, N-dimethyl-Llysine in recombinant histones. Chem Biol 2010, 17:1072-1076.

51. Wang ZPA, Zeng Y, Kurra Y, Wang X, Tharp JM, Vatansever EC, Hsu WW, Dai S, Fang XQ, Liu WSR: A Genetically Encoded Allysine for the Synthesis of Proteins with Site-Specific Lysine Dimethylation. Angewandte Chemie-International Edition 2017, 56:212-216.

52. Lee S, Oh S, Yang A, Kim J, Soll D, Lee D, Park HS: A facile strategy for selective incorporation of phosphoserine into histones. Angew Chem Int Ed Engl 2013, 52:5771-5775.

53. Park HS, Hohn MJ, Umehara T, Guo LT, Osborne EM, Benner J, Noren CJ, Rinehart J, Soll D: Expanding the genetic code of Escherichia coli with phosphoserine. Science 2011, 333:11511154.

54. Rogerson DT, Sachdeva A, Wang K, Haq T, Kazlauskaite A, Hancock SM, Huguenin-Dezot N, Muqit $M M$, Fry AM, Bayliss R, et al.: Efficient genetic encoding of phosphoserine and its nonhydrolyzable analog. Nat Chem Biol 2015, 11:496-503.

55.* Huguenin-Dezot N, De Cesare V, Peltier J, Knebel A, Kristaryianto YA, Rogerson DT, Kulathu Y, Trost M, Chin JW: Synthesis of Isomeric Phosphoubiquitin Chains Reveals that Phosphorylation Controls Deubiquitinase Activity and Specificity. Cell Reports 2016, 16:1180-1193.

This comprehensive study illustrates how the genetic encoding of PTM can enable insights into the substrate perference of E3 ligase and deubiquitinases.

56.** Zhang MS, Brunner SF, Huguenin-Dezot N, Liang AD, Schmied WH, Rogerson DT, Chin JW: Biosynthesis and genetic encoding of phosphothreonine through parallel selection and deep sequencing. Nat Methods 2017, 14:729-736.

57.** Luo X, Fu G, Wang RE, Zhu X, Zambaldo C, Liu R, Liu T, Lyu X, Du J, Xuan W, et al.: Genetically encoding phosphotyrosine and its nonhydrolyzable analog in bacteria. Nat Chem Biol 2017, 13:845-849. 
These two studies demonstrate the genetic encoding of the central PTMs phosphothreonine and phosphotyrosine.

58.* Walker OS, Elsasser SJ, Mahesh M, Bachman M, Balasubramanian S, Chin JW: Photoactivation of Mutant Isocitrate Dehydrogenase 2 Reveals Rapid Cancer-Associated Metabolic and Epigenetic Changes. J Am Chem Soc 2016, 138:718-721.

Photoactivation of a mutant form of IDH2 revealed the sequence of events following the production of the onco-metabolite (R)-2-hydroxyglutarate.

\section{Figure Legends}

Figure 1. Use of UV-activatable UAAs in the study of epigenetic processes. A Unnatural amino acids are encoded in response to UAG (amber) stop codons by the genetic addition of an aminoacyl-tRNA synthetase/tRNA $A_{\text {CUA }}$ pair to the host system. Here exemplarily shown is the encoding of UV-activatable crosslinker amino acids in histones of budding yeast. The synthetase is engineered to charge the tRNA with a particular amino acid which is then incorporated into the growing polypeptide chain by the ribosome. UV-crosslinker containing histones are integrated into the natural chromatin landscape by endogenous processes where they can be induced to form crosslink products with interacting proteins. B Trapping of a transient interaction of the Paf1C subunit Rtf1 with ubiquitin E2 conjugase Rad6 in vivo. This interaction is critical for coupling transcription by RNA polymerase-2 to H2B ubiquitination and had escaped identification by conventional methods [13*]. C Three novel UAAs that may facilitate MS analyses of crosslink products [18*,21-23]. Novel functionalities are highlighted in red.

Figure 2. Genetic encoding of lysine acetylation. A Structures of lysine (K) and NE-acetyl-lysine (AcK). B TIP60-Aurora B signalling pathway ensures robust chromosome segregation [34*]. Aurora B is activated by phosphorylation of T232 in its activation loop. The CDK1-cyclin B complex phosphorylates the acetyltransferase TIP60 that can in turn stabilize the active phosphorylated Aurora B form via acetylation of K215. C Incorporation of AcK at a single K position in GFP (or mCherry) prevents chromophore maturation. Deacetylation by sirtuins such as cobB, SIRT1 and SIRT2 allows maturation, enabling monitoring of intracellular sirtuin activity by fluorescence [37**]. 
Figure 3. Genetic encoding of alternative lysine acylations. A Structures of post-translational $N \varepsilon$ acylated lysines. B Crystal structure of the MOZ DPF binding pocket (grey surface) with accommodated Crk (orange sticks) [48]. Key residues of the MOZ DPF are shown as grey sticks. Binding of CrK is assisted by water-mediated hydrogen bonds (dashed red lines) with the amide group of CrK. C Genetic incorporation of azidonorleucine into proteins followed by site-specific lysine acylations via a traceless Staudinger ligation [49*]. The use of phosphinothioesters with varying acyl substituents enables installation of a broad range of acylation types in vitro. As a side product, azidonorleucin is reduced to lysine.

Figure 4. Photo-activation of an onco-enzyme drives cancer-associated epigenetic changes. An R172K mutation in isocitrate dehydrogenase-2 (IDH2) is associated with many types of cancer where it drives the formation of the onco-metabolite (R)-2-hydroxyglutarate. By replacing this lysine residue with a photo-activatable derivative it was possible to quantify the rate of changes in hydroxymethyl-cytosine levels affected by inhibition of ten-eleven-translocation (TET) enzymes [58*]. 


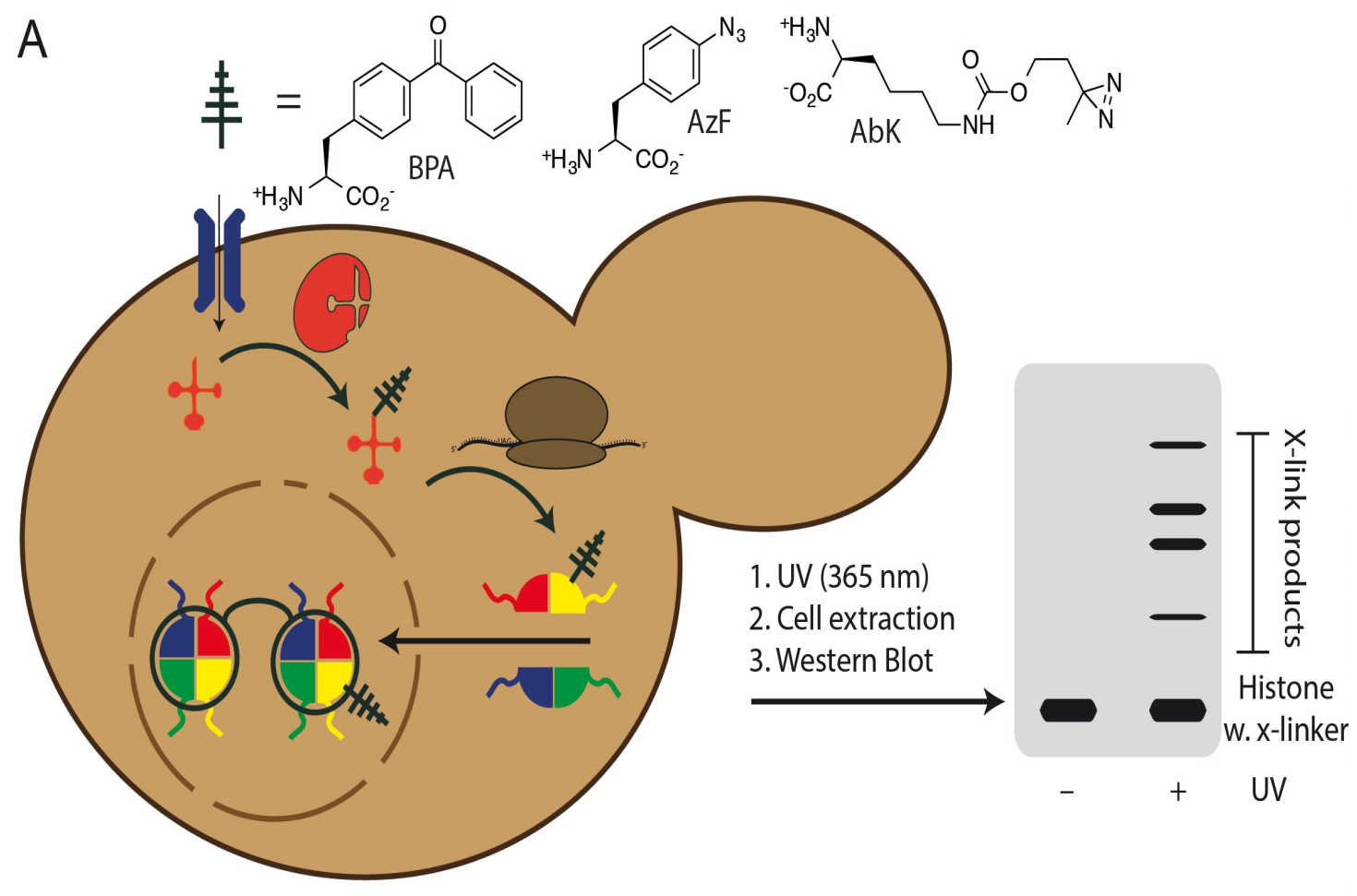

B

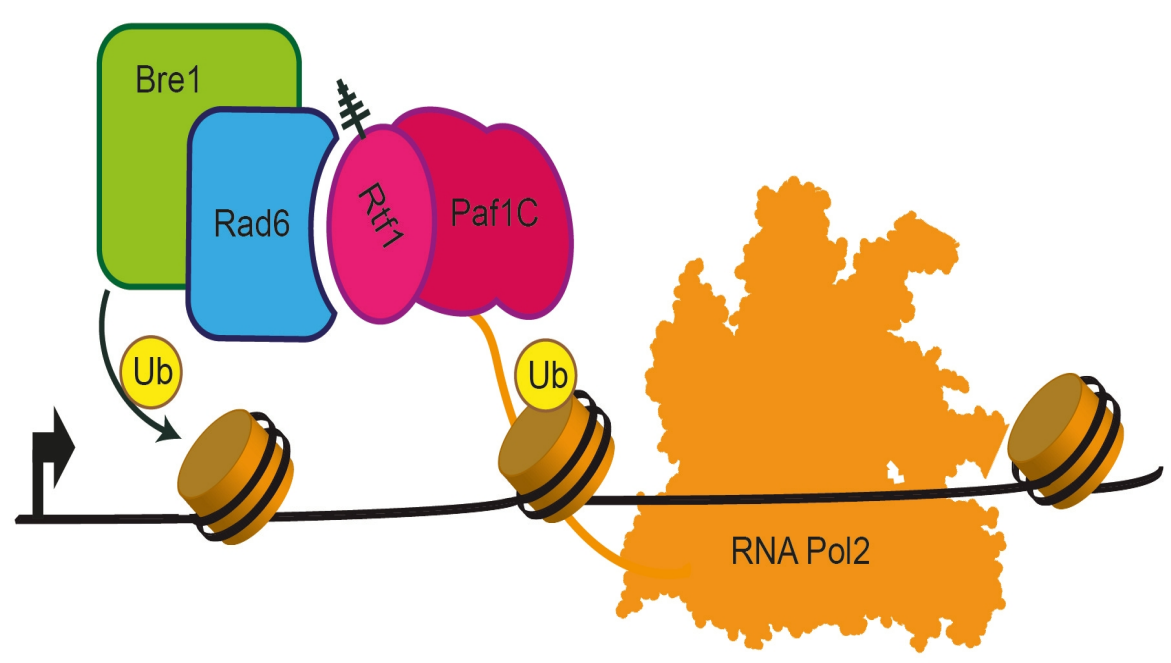

C

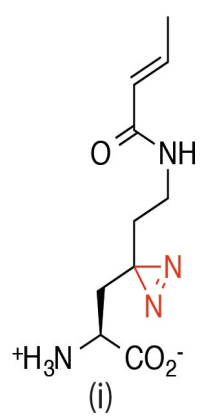<smiles>C#Cc1ccc(C(=O)c2ccc(CC(NC)OC(=O)O)cc2)cc1</smiles><smiles>CC(N)CCOC(=O)NCCCOC(=O)C(C)N</smiles>

Figure 1 
(a)

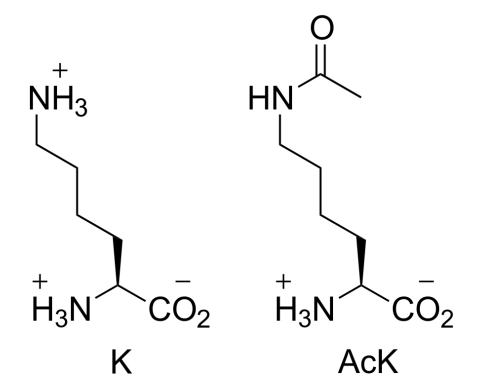

(c)

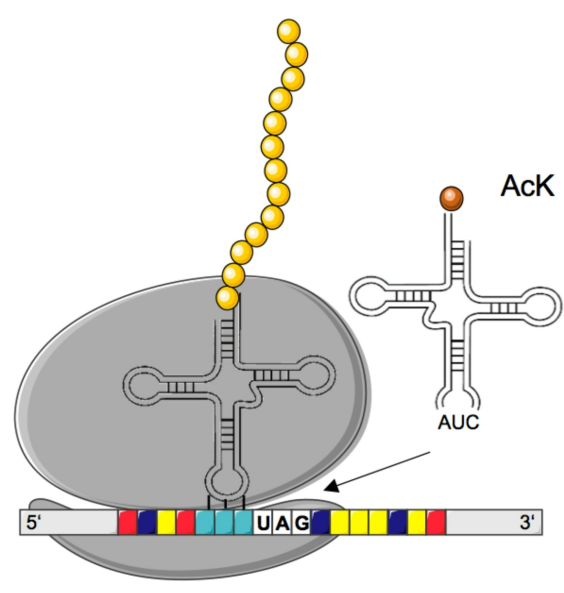

(b)

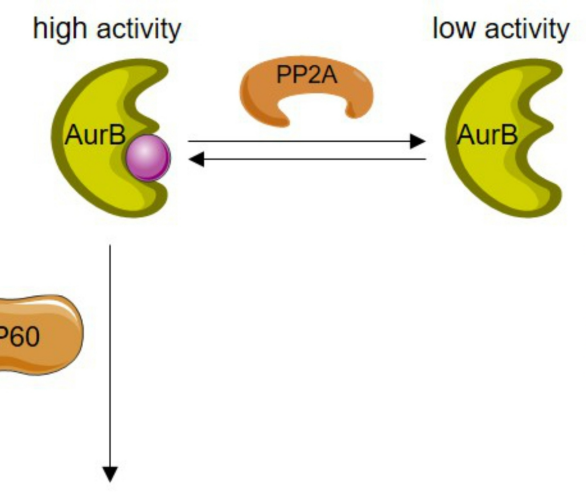

1 Acetylation
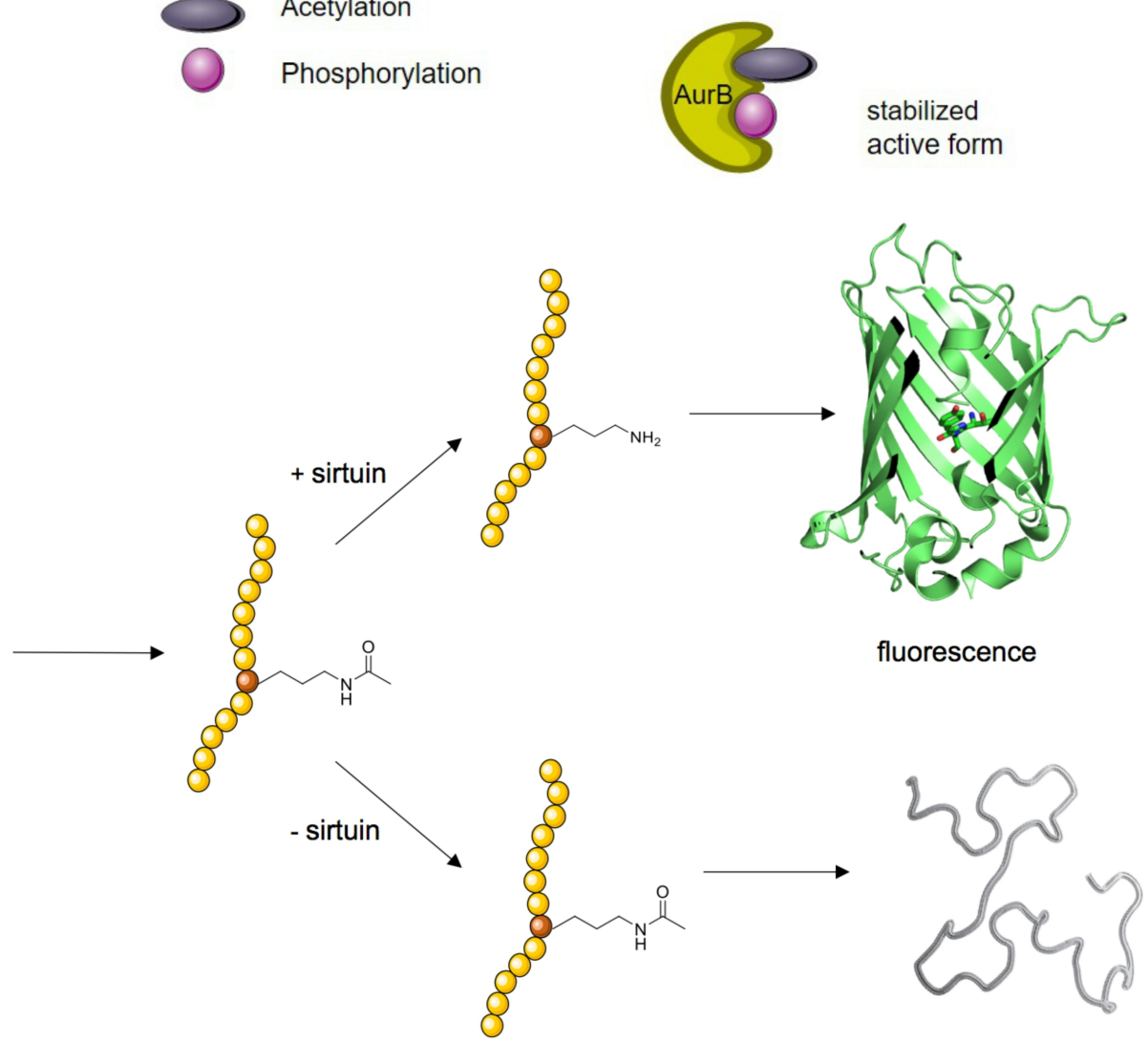

no fluorescence 
(a)

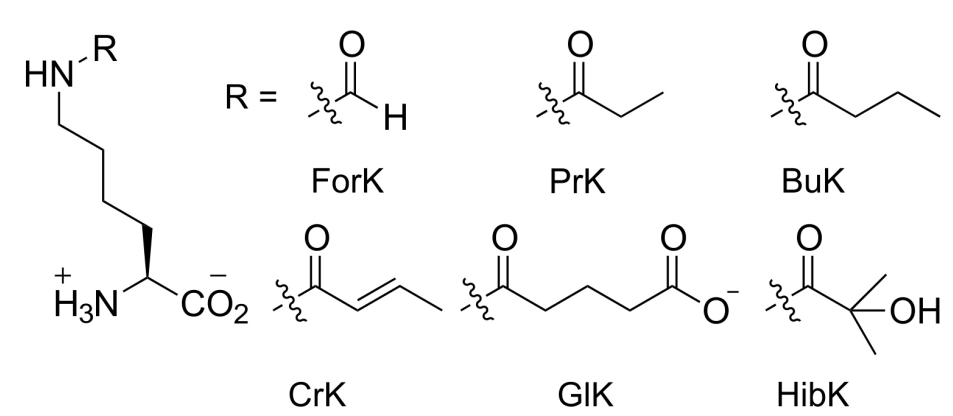

(c)<smiles>[3H][13C](=O)CC(=O)[O-]</smiles>

MaK<smiles>CC(=O)CCC(=O)[O-]</smiles>
SuK<smiles>[Y6]C(=O)CCCC[C@H]1SC[C@H]2NC(=O)N[C@H]21</smiles>

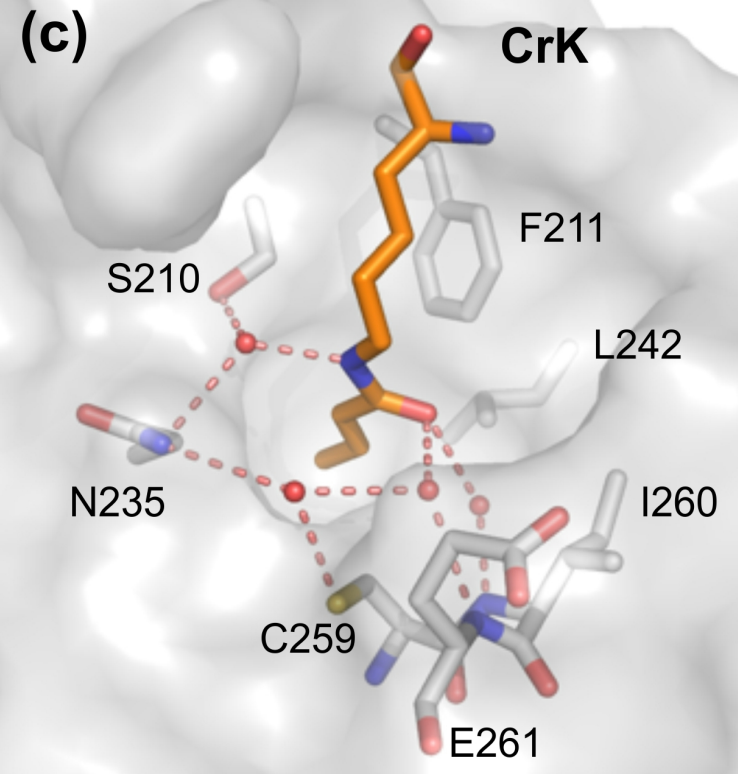

(c)

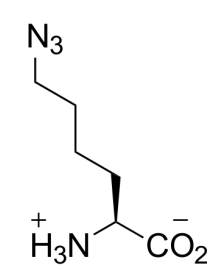

Azidonorleucin

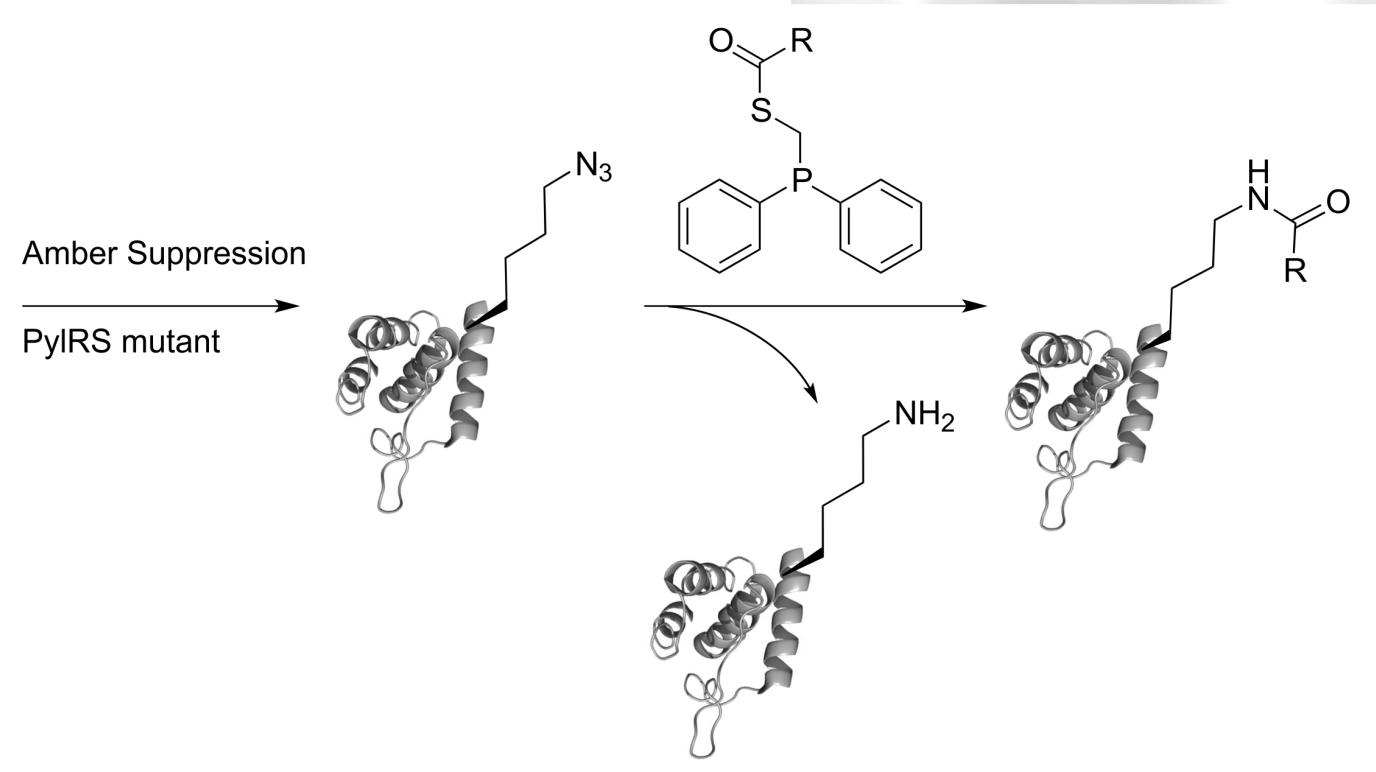




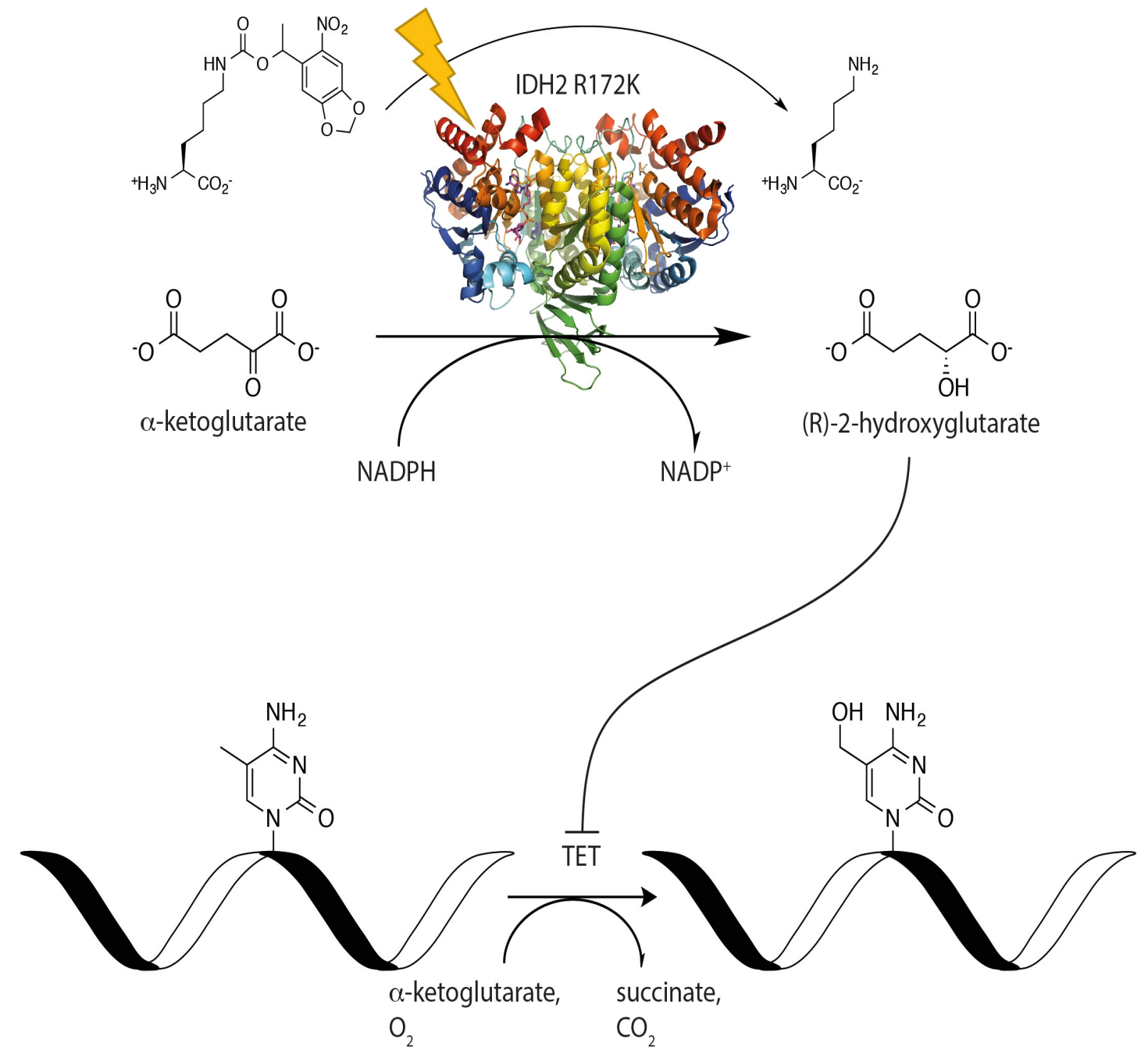

Figure 4 